\title{
ESTRUTURA E MODELO DO ACESSO A MEDICAMENTOS NO BRASIL: UMA ANÁLISE DO PROGRAMA FARMÁCIA POPULAR DO BRASIL
}

RESUMO: Este artigo analisa a estrutura do Programa Farmácia Popular do Brasil (PFPB), tendo por base as dimensões propostas no modelo da Organização Mundial da Saúde em conjunto com a Management Sciences for Health. Discute conceitos e abordagens do papel do Estado na perspectiva do desenvolvimento econômico, modelos de acesso a medicamentos e indicadores do setor saúde no contexto do desenvolvimento econômico no Brasil. Ao caracterizar o PFPB, identificaram-se elementos alinhados às dimensões disponibilidade, aceitabilidade, acessibilidade geográfica e poder de compra. A estratégia do Programa Aqui tem Farmácia Popular (PAFP) mostrou-se ser econômica, justificando manter-se como estratégia prioritária do Estado. Entretanto, há limitações a serem consideradas para que tenha melhor desempenho no que se propõe, em especial, em relação à dimensão do poder de compra da farmácia privada.

PALAVRAS-CHAVE: Desenvolvimento Econômico, Acesso a Medicamentos Essenciais, Programa Aqui tem Farmácia Popular.

\section{INTRODUÇÃO}

A Assembleia Mundial de Saúde aprovou, no ano de 1975, o conceito de medicamentos essenciais, promovendo a utilização de listas desses medicamentos e a implementação de uma política nacional, principalmente em países de baixa e média renda. São quatro os fatores críticos apontados pela Organização Mundial da Saúde (OMS) para a melhoria do acesso: a seleção racional de medicamentos e desenvolvimento de produtos; os preços acessíveis a governos e consumidores; o financiamento sustentável através de arrecadações governamentais gerais e do seguro social; e o sistema de abastecimento confiável com um mix de serviços públicos e privados (WORLD HEALTH ORGANIZATION - WHO 2000).

Estimativas do número de pessoas com acesso aos medicamentos essenciais quase dobraram entre 1977 e o final dos anos 1990 através de uma combinação entre fornecimento público e privado (QUICK; HOGERZEIL 2002² apud BIGDELI et alii 2012). Apesar do êxito, os principais resultados desejados desse tipo de acesso são o uso racional $^{3}$ e a satisfação do usuário com o serviço de saúde (BERMUDEZ et alii 2004), os quais ainda permanecem com limitações entre as populações mais pobres e vulneráveis.

Peters et alii. (2008 ${ }^{4}$ apud BIGDELI et alii 2012) definem acesso como o uso oportuno dos serviços de acordo com as necessidades. As barreiras ao acesso podem originar-se por demanda e por oferta (ENSOR e COOPER 20045 apud BIGDELI et alii 2012). As restrições pela demanda influenciam a capacidade dos indivíduos, das famílias e das comunidades de utilizarem os serviços, enquanto as restrições de oferta são aspectos dos serviços do setor de saúde

\footnotetext{
Doutora em Economia do Desenvolvimento pelo PPGE da Universidade Federal do Rio Grande do Sul/Professora Assistente da Universidade Federal do Rio Grande do Sul do Departamento de Ciências Contábeis e Atuariais, Pesquisadora do Instituto de Avaliação de Tecnologia em Saúde - IATS e integrante do Núcleo de Empreendedorismo da UFRGS.

2 Quick JD, Hogerzeil HV. 2002. Perspectives: twenty-five years of essential medicines. Bulletin of the World Health Organization 80:913-4.

3 Conforme a Management Science For Health - MSH (1997, p. 422), “[...] há uso racional quando pacientes recebem medicamentos apropriados para suas condições clínicas, em doses adequadas às suas necessidades individuais, por um período adequado e ao menor custo para si e para a comunidade".

4 Peters DH, Garg A, Bloom G et alii. 2008. Poverty and access to health care in developing countries. Annals of the New York Academy of Sciences 1136: 161-71.

5 Ensor T, Cooper S. 2004. Overcoming barriers to health service access: influencing the demand side. Health Policy and Planning 19: 69-79.
} 
que dificultam sua obtenção. As mais importantes barreiras ao acesso a medicamentos, ainda remanescentes, podem ser identificadas por quatro dimensões: acessibilidade geográfica e financeira; disponibilidade; aceitabilidade e qualidade (PETERS et alii 2008 apud BIGDELI et alii 2012).

No Brasil, a Constituição Federal (BRASIL 1988) determina, no seu artigo 196, que a saúde é direito de todos e dever do Estado e que este direito deve ser garantido por políticas sociais e econômicas com acesso universal e igualitário. Esse direito constitucional foi regulamentado pela Lei $\mathrm{n}^{\circ} 8.080$ (BRASIL 1990) e estabeleceu o Sistema Único de Saúde (SUS) para garantir assistência terapêutica integral, inclusive a farmacêutica. Em relação à forma de acesso aos medicamentos essenciais, atualmente, a estrutura existente no Brasil, que conta com financiamento total ou parcial do governo federal apresenta, pelo menos, três alternativas: Programa Farmácia Básica (PFB) do SUS; Programa Farmácia Popular do Brasil (PFPB), através de farmácias da rede própria apoiadas pela Fundação Oswaldo Cruz (Fiocruz) e PFPB, através de farmácias privadas conveniadas ao Programa Aqui tem Farmácia Popular (PAFP). Este aproveita a dinâmica da cadeia farmacêutica já estruturada (produção-distribuição-varejo), utilizando-a para pontos de distribuição de medicamentos essenciais.

Desta forma, o acesso aos medicamentos essenciais é uma questão inserida na atenção e nos cuidados de saúde e sua perda ou sua falta é uma agressão aos direitos humanos. Sua implementação envolve uma rede de atores, públicos e privados, que desempenham diferentes papéis em função do contexto econômico, político e social dos diversos países. Neste enfoque, para que uma política pública de saúde seja avaliada em relação aos impactos e oportunidades que traz à população, torna-se necessário ter por base um modelo que identifique e avalie as relações entre as dimensões e os agentes participantes do sistema de saúde.

Assim, tratando-se de uma ação estratégica no escopo da política de assistência farmacêutica no país, este artigo tem por objetivo analisar a estrutura do $\mathrm{PFPB}$, tendo por base as dimensões propostas no modelo da OMS em conjunto com a Management Sciences for Health (MSH) (WHO e MSH 2000). Fundamentalmente, as informações que sustentam o presente artigo resultam do processo de revisão teórica na literatura específica em mídias impressas (livros, revistas e publicações de instituições federais) e virtuais (sites, blogs, etc.).

O artigo está estruturado em cinco seções. Esta introdução que contextualiza o tema do artigo; seguida da seção que apresenta conceitos e abordagens em relação ao papel do Estado na perspectiva do desenvolvimento econômico e breve discussão de indicadores, reforçando a importância do setor saúde para o desenvolvimento econômico no Brasil. A terceira seção apresenta alguns modelos de acesso medicamentos. Na quarta seção analisa-se as propostas de acesso a medicamentos, implementadas pelo governo federal através do PFPB, mais especificamente, do PAFP, fundamentado no modelo WHO e MSH (2000) e as considerações finais que encerram a análise das informações em relação ao que se propunha para o texto, indicando limitantes e potencialidades que o PAPF apresenta para o desenvolvimento econômico do país.

\section{DESENVOLVIMENTO ECONÔMICO E O PAPEL DO ESTADO}

Segundo a teoria clássica, o sistema econômico seria dominado pelas leis de mercado, se auto ajustando com tendência ao pleno emprego e ao laissez-faire de Adam Smith. Embora para Corazza (1985), Smith deixa transparecer que o Estado ocupa uma ampla função na economia, por exemplo, na administração da justiça é ele que deve combater o corporativismo.

Corazza (1985), em relação à Teoria de Ricardo, baseada na autorregulação dos mercados, expõe que o Estado não está tão ausente das preocupações do autor na medida em que exerce papel importante quando os mecanismos de regulação falham, ameaçando a interrupção do processo de acumulação através dos obstáculos naturais. 
Destaca-se, neste contexto, a denominada Escola Keynesiana ou Keynesianismo, teoria econômica consolidada pelo economista inglês Jonh Maynard Keynes em seu livro General Theory of Employment, Interest and Money (KEYNES 1936). Sua síntese influenciou a renovação das teorias econômicas clássicas, bem como a reformulação da política de livre mercado. A concepção desta teoria consiste, basicamente, na organização político-econômica, fundamentada na constituição do Estado como agente indispensável de controle da economia. Segundo Keynes (2004), a mais importante agenda do Estado relaciona-se às funções que estão fora do âmbito do indivíduo, cujas decisões ninguém assume se aquele não o fizer.

Da mesma forma, Myrdal (1968) defende que as regulamentações sociais, através da intervenção estatal diferem das antigas políticas de auxílio à pobreza, em que os investimentos eram considerados gastos, não estando relacionados ao aumento do nível de produtividade, embora vitais para o desenvolvimento econômico de um país. Para o autor é fundamental e indispensável a ação estatal para controlar as forças do mercado.

Pereira (1996) reforça, no contexto brasileiro, a importância da intervenção do Estado uma vez que a reforma pública das funções deste está apoiada na proposta de administração pública gerencial, como resposta à grande crise dos anos 80 e à globalização da economia. Para o autor, seu papel é o de facilitar que a economia nacional torne-se, internacionalmente, competitiva. Ademais, a regulação e a intervenção são necessárias na educação, na saúde, na cultura, no desenvolvimento tecnológico e nos investimentos em infraestrutura.

Neste sentido, Reis e Bermudez (2004) apontam que as reformas do Estado, pressionadas pela globalização, direcionam a formulação de políticas que busquem garantir equidade nas ações de saúde, reforçando o papel do Estado no contexto das reformas atuais e na questão do acesso a medicamentos. Segundo os autores, a complementaridade entre o setor público e o setor privado tem por objetivos: garantir a equidade no acesso a medicamentos, prioritariamente, aos medicamentos essenciais; assegurar a eficiência no uso de recursos para medicamentos; e promover o consumo racional dos medicamentos em ambos os setores, assegurando o cumprimento das normas de qualidade.

Para Rodrik (2005), em seu trabalho Growth Strategies, a definição de estratégias de crescimento é o conjunto de políticas econômicas e arranjos institucionais direcionados aos padrões de vida das populações dos países desenvolvidos. Um dos pontos constatados refere-se às especificidades das políticas que estimulam o crescimento em determinadas economias, especialmente, no que diz respeito à limitação de estratégias para melhorias.

Segundo Nota Técnica n ${ }^{0} .12$ da Consultoria de Orçamento e Fiscalização Financeira da Câmara dos Deputados - CONOF/CD (BRASIL, 2013), em 2011, dados do Banco Mundial, indicavam que o dispêndio público e privado em saúde, em relação ao Produto Interno Bruto (PIB), foi de 9,3\% na Inglaterra, 9,4\% na Espanha, 11,2\% no Canadá e 9,0\% na Austrália. No Brasil, que possui cobertura universal, à semelhança dos países citados, esse percentual foi de $8,9 \%$.

Ainda, a Nota Técnica menciona que, nos países desenvolvidos e em desenvolvimento, grande parte do financiamento da saúde provém de fontes públicas e a maior parte do gasto privado é realizada através de planos e seguros privados. Referencia-se que o desembolso direto é maior nos países mais pobres e que a participação do setor público ganha maior importância no financiamento da saúde em países que adotam sistemas de cobertura universal (FIOCRUZ et alii 2012). De acordo com os Indicadores de desenvolvimento mundial do Banco Mundial a participação de fontes públicas no financiamento da despesa total dos sistemas no ano de 2011 variou de 68,5\%, na Austrália a 82,7\%, na Inglaterra, com uma média de 70\% e no Brasil esse percentual indicou que 45,74\% do total de despesas públicas foram aplicadas em saúde (THE WORLD BANK - TWB 2013).

Segundo o relatório de Contas Satélites de Saúde referente ao período 2007 a $2009^{6}$, a participação das atividades de saúde no valor adicionado da economia brasileira foi, em média, de 6,1\%. Nesse período, praticamente 4,5\% dos postos de trabalho e de $7,8 \%$ das remunerações do trabalho no país estavam em atividades de saúde, conforme apresenta a tabela 1 .

\footnotetext{
Segundo o IBGE, a previsão para a divulgação do relatório de Contas Satélites de Saúde do período posterior a 2009 é março de 2015.
} 
Tabela 1 - Indicadores de Participação da saúde na economia do Brasil - 2007 - 2009

\begin{tabular}{|c|c|c|c|c|}
\hline Indicadores de Saúde no Brasil & 2007 & 2008 & 2009 & $\begin{array}{l}\text { Variação } \\
\text { período }\end{array}$ \\
\hline $\begin{array}{l}\text { Valor adicionado bruto pelas atividades de saúde como percentual do valor } \\
\text { adicionado bruto total }\end{array}$ & 6,1 & 6 & 6,2 & $1,60 \%$ \\
\hline $\begin{array}{l}\text { Despesas com consumo final de bens e serviços de saúde como percentual do } \\
\text { PIB }\end{array}$ & 8,5 & 8,3 & 8,8 & $3,50 \%$ \\
\hline Despesas com consumo final de serviços de saúde como percentual do PIB & 5,4 & 5,4 & 5,6 & $3,70 \%$ \\
\hline Despesas com consumo final de medicamentos como percentual do PIB & 1,9 & 1,8 & 1,9 & $0,00 \%$ \\
\hline $\begin{array}{l}\text { Participação dos medicamentos nas despesas com consumo final de bens e ser- } \\
\text { viços de saúde }\end{array}$ & 22 & 21,9 & 22 & $0,00 \%$ \\
\hline $\begin{array}{l}\text { Participação dos serviços de saúde nas despesas com consumo final de bens e } \\
\text { serviços de saúde }\end{array}$ & 64,3 & 65,6 & 64,5 & $0,30 \%$ \\
\hline $\begin{array}{l}\text { Crescimento médio anual do consumo final de bens e serviços de saúde das fa- } \\
\text { mílias }\end{array}$ & $\cdots$ & 5,3 & 3,5 & $\ldots$ \\
\hline Crescimento médio anual do consumo final de medicamentos das famílias & $\ldots$ & 8,6 & 6,7 & $\ldots$ \\
\hline $\begin{array}{l}\text { Crescimento médio anual do consumo final de serviços de saúde da administra- } \\
\text { ção pública }\end{array}$ & $\cdots$ & 10,4 & 10,3 & $\ldots$ \\
\hline $\begin{array}{l}\text { Crescimento médio anual do consumo final de medicamentos da administração } \\
\text { pública }\end{array}$ & $\cdots$ & 28,3 & 4,9 & $\ldots$ \\
\hline
\end{tabular}

Fonte: Elaborada pela autora a partir de dados do IBGE (2012).

Os gastos correntes com saúde, equivalentes às despesas com consumo final de bens e serviços de saúde, alcançaram $\mathrm{R} \$ 283,6$ bilhões em 2009, representando um aumento de 10,1\% em relação ao ano de 2007, descontando variações de preços.

As despesas com consumo final de bens e serviços de saúde corresponderam, no período, a 8,5\% do PIB, em média, mas oscilaram de 8,3\% em 2008 a 8,8\% em 2009. O crescimento das despesas com saúde, em relação ao PIB no ano 2009 pode ser explicado pela queda deste. Com isso, os gastos com saúde passaram a mobilizar uma parcela maior do PIB.

Em 2009, o consumo de serviços de saúde movimentou, em média, 5,5\% do PIB e as despesas com consumo final de medicamentos, 1,9\%. Os medicamentos foram responsáveis por cerca de 22,0\% do total de gastos com saúde. Os serviços de saúde foram responsáveis por $64,8 \%$. As despesas públicas per capita com consumo de bens e serviços de saúde em 2007 passaram de $\mathrm{R} \$ 502,36$ para $\mathrm{R} \$ 645,27$, em 2009, o que representa um crescimento em valores correntes de 28,4\%. As despesas per capita privadas de 2007 subiram de $\mathrm{R} \$ 698,98$ para $\mathrm{R} \$ 835,65 \mathrm{em} 2009$, um aumento de $19,6 \%$ em valores correntes.

Em 2008 e 2009, o consumo de bens e serviços de saúde pelas famílias cresceu 5,3\% e 3,5\%, respectivamente, tendo crescimento médio anual do consumo final de medicamentos de $8,6 \%$ e $6,7 \%$. Nesses anos, o crescimento do consumo de bens e serviços de saúde pelo governo foi de 6,3\% e 5,2\%, havendo também uma redução no crescimento médio anual do consumo final de bens e serviços de saúde de 6,3\% em 2008 e 5,2\% em 2009.

Ainda que a participação da administração pública nas despesas com saúde, relativamente às famílias, tenha crescido no período, a participação das despesas com saúde no total das despesas do governo se manteve relativamente estável, oscilando entre $17,5 \%$ e $18,0 \%$, no período.

Viana e Elias (2007) discutem as possíveis soluções para que o Brasil constitua um modelo virtuoso de associação entre saúde e desenvolvimento. Salientam a importância do complexo produtivo desse elemento para o 
desenvolvimento nacional, por se tratar de um campo em que inovação tecnológica e acumulação de capital geram oportunidades de investimento, de trabalho e de renda, produzindo, assim, avanços importantes para melhorar o estado de saúde das pessoas. Para os autores, esse componente pode ser visto por três óticas e dessa interação são obtidas as características dos modernos sistemas de saúde. Enquanto direito, a economia alinha a saúde ao fenômeno de desmercantilização do acesso, criando os sistemas de proteção social; como bem econômico, considerando a saúde a partir da mercantilização da oferta, ocasionando diversos fenômenos como o assalariamento dos profissionais e formação das operadoras de planos e seguros saúde; e como espaço de acumulação de capital, formando o Complexo Econômico-Industrial da Saúde (CEIS), trazendo consigo a globalização financeira da riqueza.

O Brasil, nas últimas décadas, tem apresentado mudanças significativas na expectativa de vida, na redução da mortalidade infantil, no aumento de cobertura em programas de imunizações e na incorporação de tecnologias de ponta e resolutivas, evoluindo, com resultados e impactos positivos nos indicadores epidemiológicos e econômicos (BRASIL 2008), demandando políticas diferenciadas no âmbito da atenção à saúde. Esta tem sido apontada como o problema mais importante enfrentado no cotidiano $(24,2 \%)$, seguido por desemprego $(22,8 \%)$, situação financeira $(15,9 \%)$ e violência (14\%) (CONSELHO NACIONAL DOS SECRETÁRIOS DE SAÚDE - CONASS 2003). Segundo o relatório de Contas Satélites de Saúde, referente ao período 2007 a 2009 (INSTITUTO BRASILEIRO DE GEOGRAFIA E ESTATÍSTICA - IBGE 2012), a participação das atividades de saúde no valor adicionado da economia brasileira foi, em média, de 6,1\%. Em 2009, os gastos em saúde representavam 8,8\% do Produto Interno Bruto (PIB).

Pelo exposto e com o intuito de avaliar a estrutura do PFPB, proposto pelo Estado como estratégia de política pública de saúde, faz-se necessário o conhecimento de modelos de acesso a medicamentos existentes que possibilitem esta análise, a próxima subseção tem esse objetivo.

\section{MODELOS DE ACESSO A MEDICAMENTOS}

A literatura apresenta pelo menos quatro modelos de acesso para a população a medicamentos: da OMS (WHO, 2004); de Frost e Reich (20107 BIGDELI et alii 2012) e da OMS e da Management Sciences for Health (WHO e MSH 2000).

No modelo da OMS (WHO 2004) são propostas a garantia de qualidade e os sistemas de gestão para sustentar todos os componentes desse acesso. Para tanto, estabelece quatro dimensões: uso racional; preço acessível; financiamento sustentável; e sistema de saúde e abastecimento confiáveis.

O modelo de Frost e Reich (2010) analisa a forma de acesso a medicamentos pelas populações pobres, tendo como determinantes: arquitetura; disponibilidade; acessibilidade e adoção e ainda, examina as tecnologias médicas.

O modelo teórico de Bigdeli et alii (2012) é mais complexo que os anteriores, porque abrange as discussões recentes sobre o sistema de saúde, sua complexidade e seu dinamismo (PAINA; PETERS e SHEIKH et alii 2011 ${ }^{8}$; VAN OLMEN et alii $2012^{9}$ apud BIGDELI et alii 2012).

Outro modelo que a literatura propõe é o desenvolvido a partir do trabalho de Penchansky e Thomas (1981), adaptado por Luiza (2003) baseado nas proposições do Consultative Meeting em Ferney-Voltaire (WHO e MSH 2000), reconhecido como o modelo da OMS e da MSH (WHO e MSH 2000).

\footnotetext{
Sheikh K, Gilson L, Agyepong IA et alii. 2011. Building the field of health policy and systems research : framing the questions. PLoS Medicine 8: $1-6$.

8 Paina L, Peters DH. 2011. Understanding pathways for scaling up health services through the lens of complex adaptive systems. Health Policy and Planning 26: 1-9

9 Van Olmen J, Criel B, Bhojani U, Marchal B et alii. 2012. The health system dynamics framework: the introduction of an analytical model for health system analysis and its application to two case-studies. Health, Culture and Society 2: 10-21.
} 
Há mais de 30 anos, Penchansky e Thomas (1981, p.127) publicaram um artigo intitulado The concept of access: definition and relationship to consumer satisfaction e já na frase de abertura do mesmo, apontavam: "[...] acesso é uma grande preocupação em política de saúde e é uma das palavras mais usadas nas atuais discussões sobre o sistema de saúde". Passadas várias décadas esta preocupação ainda persiste. A figura 1 sintetiza o sistema de intervenções proposto pelo modelo WHO e MSH (2000), cujo núcleo central é o uso racional dos medicamentos e a satisfação do usuário.

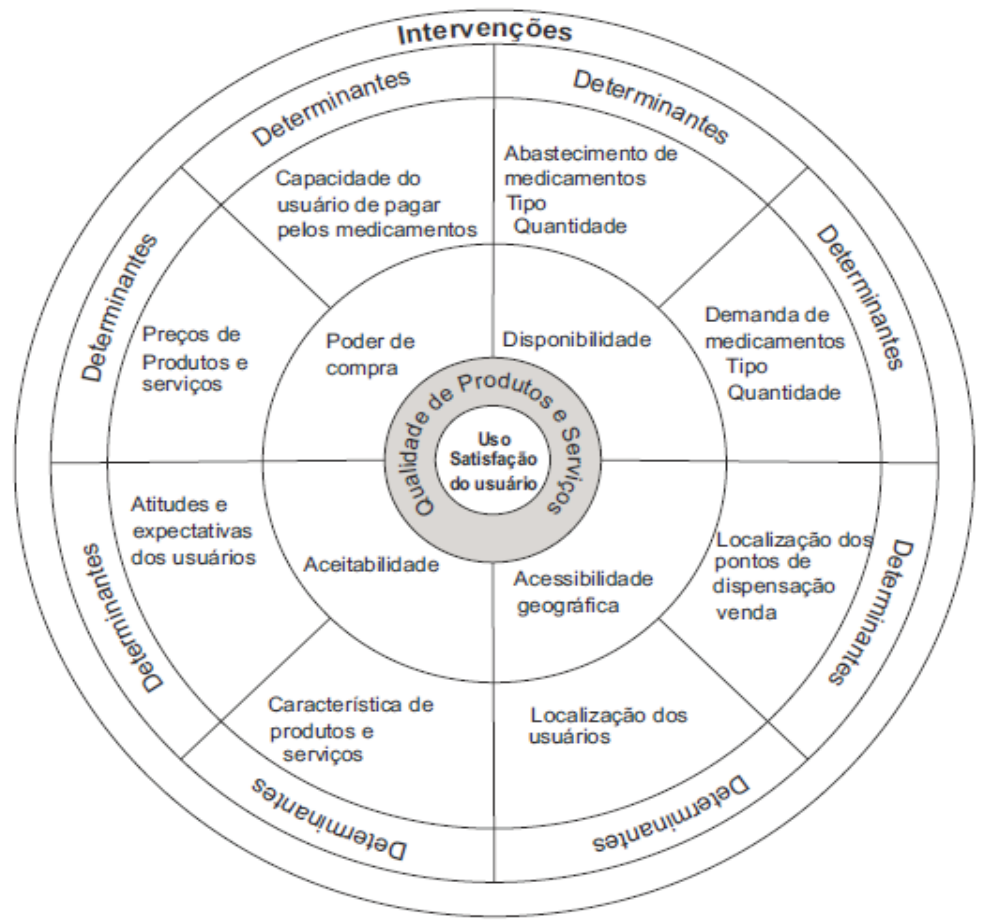

Figura 1. Modelo de acesso a medicamentos Fonte: Adaptada por Luiza (2003) a partir de WHO e MSH (2000).

A qualidade de produtos e serviços é o elemento transversal do modelo e se constitui num componente essencial do acesso, perpassando as quatro dimensões identificadas: disponibilidade; aceitabilidade; acessibilidade geográfica e poder de compra ${ }^{10}$, tendo cada uma delas, dois determinantes específicos. Cada dimensão do modelo WHO e MSH (2000) tem suas próprias características. A qualidade de produtos e serviços consiste na aplicação da ciência e da tecnologia médicas de modo que resulte o máximo de benefícios para a saúde sem aumentar, com isto, seus riscos (DONABEDIAN 1984).

Considerando a forma de provisão dos medicamentos, pública ou privada, gratuidade ou copagamento, o passo para garantir seu acesso está em sua disponibilidade no momento em que o usuário dele necessitar, na quantidade necessária e em condições de qualidade adequadas ao uso. A dimensão da disponibilidade é então determinada pelo abastecimento e pela demanda de medicamentos (tipo e quantidade).

Cabe mencionar que um sistema de abastecimento organizado requer um adequado e também, um bom modelo de distribuição, que garanta tanto a qualidade dos produtos ao longo de toda a cadeia estruturada, quanto a disponibilidade regular, conforme as necessidades específicas e a capacidade instalada dos serviços de saúde em cada localidade. Deve também incluir ações que promovam a qualidade dos serviços e produtos oferecidos no momento da dispensação, ocasião que há contato direto dos profissionais do sistema com o usuário.

\footnotetext{
$\overline{10}$ O termo poder de compra foi traduzido por Luiza (2003) a partir da expressão original affordability, qualidade de ser financeiramente possível.
} 
Uma segunda dimensão do modelo é a acessibilidade geográfica, definida pela relação entre os pontos de dispensação e venda e a localização dos seus usuários. Sabendo-se que podem coexistir diferentes mecanismos para obtenção de medicamentos, é importante que cada um deles esteja disponível à sua clientela alvo, considerando a distância e o tempo de viagem entre a localização do usuário e o ponto de distribuição dos medicamentos.

A dificuldade de acesso aos medicamentos essenciais constitui um problema, sendo necessário priorizar a qualidade de serviços e de produtos oferecidos: medicamentos, treinamento contínuo e a disseminação de informações. Os subsistemas do ciclo da assistência farmacêutica e do mercado de medicamentos englobam: política nacional; desenvolvimento de medicamentos; registro e regulação; produção/importação; distribuição atacadista; informação de medicamentos; prescrição/propaganda; dispensação/venda varejista e consumo pela população. Assim, estabelece-se uma relação complexa entre os atores envolvidos: setor público, setor privado não lucrativo e setor privado lucrativo (BENNETT; QUICK e VELÁSQUEZ $1997^{11}$ apud BIGDELI et alii 2012). A proposta desta interação apresentada pelos autores está ilustrada no Anexo C.

A terceira dimensão do modelo de acesso em análise é a aceitabilidade de produtos e serviços oferecidos quanto à sua adequação às características, necessidades e expectativas do usuário, assim como ao atendimento dos padrões técnicos de boas práticas de funcionamento. Elementos como: organização do serviço; tempo de espera para atendimento; conforto de atendimento; cortesia e orientação quanto ao uso dos medicamentos são aspectos fundamentais que devem ser medidos para avaliação da satisfação do paciente com os serviços oferecidos.

O poder de compra é uma importante dimensão do acesso, estando presente em todas as abordagens. É a mais crítica dentre todas, tornando-se ponto central do atual debate sobre o tema. Representa a adequação entre o preço e a capacidade aquisitiva dos usuários em cada mercado, bem como representa as diferentes formas de financiamento existentes, as quais se constituem importantes aspectos a serem considerados quando se discute acesso a medicamentos.

Uma vez que o poder de compra tem por determinantes a capacidade de pagar pelos medicamentos e os preços de produtos ou serviços, as políticas públicas nesta área objetivam, principalmente, reduzir o valor de compra dos medicamentos ou então, de atuar nos seus mecanismos de financiamento, favorecendo o comprador, seja ele público ou privado. Em consequência há redução das barreiras ao acesso.

Dentre os fatores que determinam o poder de compra destaca-se a limitada concorrência, oriunda das falhas de mercado, cujos principais motivos são: existência de oligopólios e de monopólios; proteção por patentes e lealdade a marcas; assimetria de informação; e separação das decisões sobre prescrição, consumo e financiamento (MADRID; VELÁSQUEZ e FEFER 1998; RÊGO 2000).

A divulgação, de forma regular e confiável, dos preços praticados por diferentes fornecedores a diferentes compradores, nacional ou internacionalmente, pode ser uma ferramenta importante para orientar as aquisições, tornando o processo mais transparente e melhorando a capacidade de negociação e de planejamento dos compradores (MÉDECINS SANS FRONTIERES - MSF 2002; WHO 2004; MSH 2004), possibilitando, no caso de financiamento pelo Estado, melhor gestão dos recursos aplicados.

A prática de preços diferenciados, em função dos diversos níveis de poder de compra de governos, tem sido defendida como viável tanto comercial quanto politicamente, até porque, os países que têm maior necessidade do uso desta prática, são também aqueles com menor participação, em valor financeiro, no mercado farmacêutico (WHO e WORLD TRADE ORGANIZATION SECRETARIATS - WTO 2001).

Em alguns países, as tarifas alfandegárias, os impostos de comercialização e as margens de lucro praticadas pelos atacadistas e varejistas podem aumentar bastante os preços a serem pagos pelo comprador, reforçando ainda mais

\footnotetext{
11 BENNETT, S; QUICK, J. D.; VELÁSQUEZ, G. Public-private roles in the pharmaceutical sector: implications for equitable acess and rational drug use. [S.1.]: WHO, 1997. (Health Economics and Drugs, 5).
} 
que estes fatores também devem ser objeto de atenção dos governos durante a formulação de suas políticas públicas.

Por fim, o atendimento às quatro dimensões do modelo WHO e MSH (2000), disponibilidade, acessibilidade geográfica, aceitabilidade e poder de compra, possibilita que diferentes estratégias e ações no âmbito da saúde possam contribuir para a melhoria de acesso da população aos medicamentos essenciais, garantindo, a partir do dever do Estado, o cumprimento do direito dos cidadãos. As dimensões do modelo WHO e MSH (2000) assemelham-se às atuais características do PFPB, estratégia de política pública de assistência farmacêutica adotada pelo governo federal brasileiro para ampliação do acesso aos medicamentos essenciais.

Uma forma de avaliar políticas públicas de saúde de uma nação é através da medição e acompanhamento dos seus agregados econômicos. Estes devem evidenciar as características do que está sendo avaliado, traduzindo os conceitos considerados na política, seu contexto e seus efeitos esperados, dentro de uma medida específica possível de ser interpretada. Possibilitam subsidiar a formulação, implementação e acompanhamento dessas políticas por fornecer, aos tomadores de decisões, informações sobre o setor quanto aos empregos gerados, tamanho das indústrias de medicamentos, fármacos, materiais, equipamentos médicos e produção de serviços de saúde públicos e privados. Com o objetivo de contextualizar a situação brasileira, a seção a seguir apresenta uma breve análise quanto à importância da relação saúde e desenvolvimento econômico no Brasil, a partir da análise de seus principais indicadores.

As políticas públicas voltadas à criação de Programas para a ampliação do acesso aos medicamentos essenciais procuram melhorar indicadores sociais dentre eles, o aumento de renda, de postos de trabalho, de produtividade, de oferta de serviços privados e públicos, de produção da indústria farmacêutica e ainda, quando há financiamento do governo, buscam reduzir os custos com internações e os gastos familiares com este tipo de bem e serviço.

Assim, para que isto ocorra, é recomendável que se tenha por base um modelo de acesso. Sob esta perspectiva, a próxima seção apresenta informações quanto à evolução, características e estrutura do PFPB, descrevendo semelhanças do Programa às quatro dimensões do modelo de acesso aos medicamentos essenciais, proposto pela WHO e MSH (2000): disponibilidade; acessibilidade geográfica; aceitabilidade e poder de compra, cada qual com seus determinantes.

\section{ESTRUTURA DA ASSISTÊNCIA FARMACÊUTICA NO BRASIL}

Para ter acesso a medicamentos na atenção básica do SUS, através do PFB do SUS, o indivíduo precisa ser atendido em uma Unidade Básica de Saúde (UBS) ou hospital público com prescrição do médico e de acordo com portarias e protocolos elaborados pelos entes públicos. Os itens da Relação de Medicamentos Essenciais (RENAME) estão disponíveis nas farmácias do SUS para o usuário, gratuitamente.

Com o objetivo de promover a ampliação do acesso aos medicamentos essenciais, em junho de 2004, a Política de Assistência Farmacêutica criou o PFPB. Em seu lançamento, os medicamentos eram disponibilizados a baixo custo, através do Sistema de Copagamento, posteriormente, em 2010, alguns passaram a ter gratuidade.

Como justificativas da criação do PFPB pode-se considerar que, por um lado, contribui na redução do impacto do orçamento familiar causado pela compra de medicamentos, permitindo a compra sem interrupção no tratamento por questão financeira; por outro, permite reduzir os gastos do SUS com as internações que são provocadas pelo abandono e/ou falta do tratamento. Constitui-se, igualmente, argumento para sua implementação o fato de que são oferecidos medicamentos para tratamento de doenças com maior incidência no país. Segundo o Ministério da Saúde (MS), o Programa foi estabelecido sem prejuízo das ações de suprimento já previstas e garantidas pelo SUS, caracterizando-se como uma nova política de assistência, dentro do Sistema Único. Entretanto, segundo Silva Filho (2010) a instituição do PFPB fere os princípios da universalidade, equidade e integralidade, uma vez que o Sistema de Copagamento gera 
discriminação social com aqueles que não possuem condições de comprar o medicamento e que o governo teria a obrigação de distribuir gratuitamente, conforme rege a Carta Magna.

O PFPB realiza uma atividade que antes de sua criação era praticada apenas pela iniciativa privada, a despeito de que seja um serviço de interesse público. Assim, o Programa está inserido numa política maior do governo federal, a Política de Assistência Farmacêutica.

Na concepção do PFPB foi definido que a gestão das compras dos medicamentos, abastecimento das unidades e a capacitação dos profissionais que atuam nas unidades seriam de responsabilidade da Fiocruz com os recursos da união, e os gastos operacionais com pessoal, de infraestrutura e manutenção seriam de responsabilidade dos municípios brasileiros.

Pretendia-se com PFPB, a construção de farmácias populares com financiamento integral do governo federal. A meta, no decorrer de cinco anos subsequentes à sua criação, era construir mais de 20 mil instalações distribuídas em todas as unidades federativas do país. Passados dois anos, em 2006, como o crescimento dessas não correspondia às expectativas, o governo federal determinou uma nova estratégia para ampliação e facilidade de acesso aos medicamentos essenciais, implantando o Programa Aqui tem Farmácia Popular.

Os principais argumentos para a criação do PAFP foram: utilização da estrutura e pontos de distribuição das farmácias privadas; redução do gasto com construção de novas instalações de farmácias populares (gerenciadas pela Fiocruz); complementaridade aos outros dois Programas de distribuição de medicamentos (unidades próprias e farmácias básicas do SUS); e a ampliação do acesso para maior parte da população.

O PAFP constitui-se de um convênio do governo federal com farmácias e drogarias privadas, aproveitando a dinâmica da cadeia farmacêutica (produção-distribuição-varejo). A estratégia da parceria do governo federal com o setor privado varejista farmacêutico, com a utilização de pontos de distribuição de medicamentos essenciais já estruturados, apresentou benefícios de tempo para aceleração da ampliação do acesso.

A partir desta nova estratégia, o PFPB passou a atuar com dois eixos de ação:

a) Através de uma rede própria de farmácias populares em parceria com municípios, gerida pela Fiocruz; e

b) Através de uma parceria com farmácias e drogarias da rede privada, denominada PAFP.

Para participar do PAFP, as farmácias privadas solicitam o convênio junto ao MS e com a aprovação, passam a disponibilizar o acesso aos medicamentos essenciais, previamente definidos em uma lista específica, contemplando 40 itens da RENAME (fraldas geriátricas e 39 medicamentos) (Anexo A).

Dados recentes do governo federal publicados no Blog da Saúde (BRASIL 2014), demonstram que nos últimos três anos, o MS investiu mais de R\$3,9 bilhões no PFPB. O volume dos recursos investidos tem crescido, significativamente, neste período. Em 2011, esse investimento foi de R $\$ 774$ milhões; passou em 2012 para R \$ 1,3 bilhão e em 2013 registrou $\mathrm{R} \$ 1,9$ bilhão. O orçamento previsto para o ano de 2014 é cerca de $\mathrm{R} \$ 2,6$ bilhões.

Em 2012 o Programa contava com 572 farmácias vinculadas à Fiocruz e com 25.122 farmácias conveniadas ao PAFP. No período de 2006 a 2012 houve um crescimento de 115\% das unidades Fiocruz e de 750\% de farmácias privadas. O crescimento anual das unidades da rede própria, vinculadas à Fiocruz, apresenta um estágio estacionário e as unidades conveniadas ao PAFP cresceram, significativamente, ficando evidente que governo federal tem ampliado a rede do PFPB através do convênio com as farmácias privadas.

Segundo o Relatório de Gestão da Fiocruz (2011), em 2011 foram dispensadas 835 milhões de unidades farmacotécnicas, apresentando uma diminuição de 9,1\% com relação a 2010, em razão do crescimento de farmácias conveniadas do PAFP, ampliando de forma regional e local o acesso da população a esses medicamentos, descentralizando o atendimento pela rede própria. Outro impacto relatado foi o não funcionamento de 18 farmácias por motivos de descumprimento dos padrões operacionais logísticos e contábeis da Fiocruz.

Cabe destacar a evolução da quantidade de municípios atendidos pelo PFPB. Em 2012 havia 3.730 municípios atendidos com farmácias privadas e 441 com unidades próprias. No período de 2006 a 2012 houve um aumento de 
114\% de municípios com unidades próprias e 528\% com unidades privadas atendidas pelo Programa. No país, $67 \%$ dos municípios participam do PFPB, com unidades próprias ou privadas.

No início do PFPB todos os medicamentos eram obtidos pelo usuário pelo Sistema de Copagamento (governo financiava pelo menos $90 \%$ do valor de referência e o usuário desembolsava o restante). Em 2011, o governo promoveu a campanha A Saúde Não Tem Preço (SNTP), através da Portaria ${ }^{\circ}$ 184, definindo a gratuidade dos medicamentos da lista para os tratamentos do diabetes mellitus e da hipertensão arterial. Esta ação constituiu-se em um grande avanço. No ano seguinte, o benefício da gratuidade foi estendido aos medicamentos para o tratamento da asma. Os itens contemplados com copagamento abrangem os tratamentos de: rinite, dislipidemia, doença de Parkinson, osteoporose, glaucoma, contracepção, incluindo fraldas geriátricas.

A partir das informações apresentadas quanto à evolução, características e estrutura do PFPB reconhece-se uma semelhança do Programa às quatro dimensões do modelo de acesso aos medicamentos essenciais, proposto pela WHO e MSH (2000): disponibilidade; acessibilidade geográfica; aceitabilidade e poder de compra, cada qual com seus determinantes. Esta avaliação é feita a seguir.

Antes de se analisar o PFPB, sob este enfoque, é importante definir os elementos que serão avaliados. Dada que esta é uma política pública de assistência farmacêutica, tendo o Estado como seu principal regulador e financiador, considera-se que as dimensões devem ser vistas sob a sua perspectiva. Não esquecendo, porém, que um dos quesitos do núcleo central do modelo é a satisfação do usuário, representado pelo cidadão. Assim, as análises de algumas dimensões serão feitas sob o ponto de vista do Estado, do usuário e da farmácia privada.

A dimensão disponibilidade é determinada pelo abastecimento e pela demanda de medicamentos, em relação ao seu tipo e quantidade, alinhando-se aos seus dois determinantes. Primeiro, porque apresenta diferentes formas de provisão dos medicamentos, seja pelo eixo das unidades próprias, que conta com o apoio da Fiocruz, seja pelo eixo da parceria das farmácias e drogarias privadas. Segundo, porque aumenta a possibilidade do medicamento estar disponível.

A expansão do número de farmácias conveniadas ao Programa, em particular as farmácias privadas pelo PAFP permite envolver um universo mais amplo de atendimentos, fortalecendo a ampliação do acesso no momento em que o usuário necessitar, na quantidade necessária e em condições de qualidade adequadas. Mais farmácias, mais disponibilidade.

Segundo o modelo WHO e MSH (2000), a acessibilidade geográfica é a dimensão que diz respeito à relação entre os pontos de dispensação e de venda e a localização dos seus usuários, podendo coexistir diferentes mecanismos. Em relação a este aspecto, a estratégia de atuação do Programa, especificamente através do PAFP, demonstra atender essa dimensão, uma vez que a aprovação de novos convênios com farmácias privadas está condicionada ao atendimento da clientela alvo, especialmente em municípios e locais onde não foi possível construir farmácias populares pela rede própria.

A estratégia do PFPB pelo eixo das farmácias da rede própria, em função dos custos de construção das farmácias, bem como o tempo envolvido, mostrou não ser a melhor alternativa. O convênio com as farmácias privadas, uma vez que estas já têm a infraestrutura necessária, além de contemplar a dimensão disponibilidade, contempla a de acessibilidade geográfica, reforçando a garantia de ampliação do acesso.

A terceira dimensão do modelo de acesso proposto pela WHO e MSH (2000) é a aceitabilidade. Esta refere-se ao ajuste entre as características dos produtos e serviços e as atitudes e expectativas dos seus usuários, bem como a questão das normas técnicas e legais dos produtos e serviços oferecidos. Com os dados crescentes de atendimentos sugere-se que há o atendimento da aceitabilidade do Programa, pelos dois eixos de atuação.

O poder de compra é a quarta dimensão a ser considerada e analisada quando da elaboração de uma política pública em saúde e, em especial, a da elaboração de estratégias de atuação. Tem por determinantes a capacidade de 
pagar pelos medicamentos e os preços de produtos ou serviços. Envolve diferentes atores (setor público, setor privado não lucrativo e setor privado lucrativo). Representa a adequação entre o preço e a capacidade aquisitiva dos usuários em cada mercado, bem como, as diferentes formas de financiamento existentes. Portanto, as políticas nesta área, objetivam, principalmente, reduzir o preço dos medicamentos ou de atuar nos seus mecanismos de financiamento, favorecendo o comprador, seja ele público (Estado como financiador) ou privado (cidadão, usuário do medicamento ou a farmácia privada conveniada). Neste aspecto, quanto à perspectiva do comprador, é importante analisar as características do poder de compra de cada um deles e os possíveis impactos que a estratégia proporciona.

Sob a perspectiva do cidadão (usuário do medicamento), há opções de gratuidade e do Sistema de Copagamento $^{12}$, que disponibiliza medicamentos sem custo ou com baixo custo, garantindo assim que ele os obtenha, sem interrupção no tratamento. Nessas condições, o poder de compra do usuário possibilita reduzir ou eliminar ${ }^{13}$ barreiras que, por questões de preço, possam vir a prejudicá-lo. Isto ocorre porque, o Estado é o principal regulador e financiador.

A farmácia privada conveniada é a responsável pela compra de seus estoques, mesmo com o financiamento do governo federal ${ }^{14}$. Há diferenças entre o valor repassado pelo governo federal e o valor de compra do medicamento daquela. A indústria farmacêutica não tem interferência do Estado na determinação e limites de seus preços de venda às farmácias e aos distribuidores de medicamentos, sinalizando que não há grande poder de compra por parte da farmácia privada conveniada, ainda que esta deva oferecer todos os itens da lista do PAFP, e não apenas aqueles com melhores margens de lucro.

A análise do poder de compra sob a perspectiva do Estado enquanto comprador deve ser feita considerando cada uma das estratégias do PFPB: rede própria e PAFP. As unidades da rede própria, uma vez que têm a centralização de compra e distribuição de medicamentos pela Fiocruz, conseguiriam utilizar um poder de compra de medicamentos de forma mais eficiente. As compras feitas pela entidade são volumosas para abastecimento nacional, permitindo um poder de barganha no preço junto a fornecedores. Estes de boa parte ou praticamente da totalidade dos itens dispensados nas unidades próprias do PFPB, são de origem estatal e as compras são feitas por licitações. A própria Fiocruz produz medicamentos (insulinas) o que torna os custos de aquisição, significativamente menores em relação aos custos de aquisição para a rede farmacêutica privada.

Somente esta análise já permitiria inferir que o poder de compra através deste eixo é vantajoso para o Estado. Entretanto, o gasto que o governo tem com esta estratégia, não corresponde apenas aos gastos de compra dos medicamentos, envolve também, os gastos operacionais e de instalação das unidades.

Dados recentes informam que o MS gastou no ano de 2011, aproximadamente $\mathrm{R} \$ 120$ mil por unidade própria do PFPB e R \$37,7 mil por unidade do PAFP. Nas unidades próprias foram realizados mais de 11,4 milhões de atendimentos, com um custo médio de $\mathrm{R} \$ 37,74$. Já nas farmácias privadas foram realizados 34 milhões de atendimentos, com um valor médio de $\mathrm{R} \$ 22,3$ (STYLO FARMA 2012).

Os dados revelam que o PAFP, embora não tenha o Estado como comprador direto dos medicamentos apresenta-se como uma estratégia mais econômica para o mesmo. Uma justificativa plausível para o crescimento acelerado dos convênios firmados, demonstrando ser uma ação prioritária para o Estado, no que diz respeito à ampliação do acesso aos medicamentos essenciais.

Neste sentido, uma alternativa para que o governo atue de forma positiva, melhorando o poder de compra das farmácias conveniadas ao PAFP (uma vez que são elas que efetuam as compras direto com seus fornecedores) é

\footnotetext{
12 Embora seja uma ação do SUS, o usuário para alguns medicamentos ainda precisa pagar.

13 No caso de medicamentos com gratuidade.

14 O montante financeiro da distribuição é repassado pelo governo federal para a farmácia privada conveniada, em média, 60 dias após sua realização.
} 
definir uma regra de prática de preços junto aos distribuidores e a indústria farmacêutica através de sistema de pregão de preços e de licitações, semelhante à prática da rede própria gerida pela Fiocruz. Desta forma, todo o processo permitiria maior transparência, uma vez que os preços máximos de venda da indústria/distribuidor para as farmácias privadas seriam regulados e acompanhados pelo governo federal, dado que além do valor de compra do medicamento, as farmácias têm gastos operacionais adicionais e margem de lucro a serem considerados.

Esta análise sobre o poder de compra na perspectiva do Estado nas duas estratégias do PFPB, rede própria e farmácias privadas, permite apontar elementos possíveis de melhorias nas estratégias desenvolvidas. Por fim, a partir das informações coletadas e analisadas, conclui-se que o Programa Aqui tem Farmácia Popular apresenta maior semelhança às dimensões propostas pelo modelo WHO e MSH (2000) que o eixo de ação das farmácias da rede própria, embora ainda possam ser aprimoradas. Na seção a seguir são tecidas considerações finais pertinentes aos assuntos tratados neste artigo.

\section{CONSIDERAÇÕES FINAIS}

Ao caracterizar o PFPB identificaram-se elementos alinhados às dimensões disponibilidade, aceitabilidade, acessibilidade geográfica e poder de compra. De forma geral, a partir das informações coletadas e analisadas, o PAFP apresenta melhor semelhança às dimensões propostas pelo modelo WHO e MSH (2000) que o eixo das farmácias próprias gerenciadas pela Fiocruz.

Os resultados do volume investido, da extensão do benefício em termos de usuários, da aceitabilidade, da distribuição e acessibilidade geográfica da estratégia são significativos. Nos últimos três anos, o MS investiu mais de R \$ 3,9 bilhões no PFPB. Foram cerca de R R 774 milhões em 2011, R\$ 1,3 bilhão em 2012 e 1,9 bilhão em 2013. O orçamento para 2014 é cerca de $\mathrm{R} \$ 2,6$ bilhões.

Estes resultados reforçam os dados recentes publicados pelo MS no Blog da Saúde, (BRASIL, 2014) que indicam que 19,4 milhões de brasileiros foram beneficiados com medicamentos gratuitos para o tratamento de asma, hipertensão e diabetes, em 4.119 cidades com 30.146 farmácias públicas ou privadas que aderiram ao PFPB. O número de municípios mais carentes do país contemplados superou a meta traçada pelo governo federal de alcançar 1.377 municípios de extrema pobreza até o ano de 2013. Quando a campanha SNTP foi lançada, em 2011, havia 578 municípios contemplados.

O crescimento do PAFP desde a sua concepção, em 2006, alcançou mais de $750 \%$ de aumento, demonstrando sua aceitabilidade. Ao final da redação deste texto, o dado que se tinha era de que o PAFP atua em mais de 3,7 mil municípios com cerca de 30 mil farmácias privadas conveniadas. Desta forma reconhece-se que houve:

c) amplo e rápido crescimento ao acesso a medicamentos, em parte pelo aproveitamento das estruturas existentes das farmácias privadas;

d) fortalecimento da parceria do governo federal, utilizando estruturas privadas para sua função públicosocial;

e) ampliação dos estabelecimentos privados credenciados, participantes do Programa, tendo seu número dobrado em três anos, de 14 mil em 2011 a 29,6 mil em janeiro 2014;

f) ampliação das opções com diversidade de acesso a medicamentos contemplados no Programa, antes circunscritas apenas às farmácias do SUS, reforçando o atendimento à dimensão da acessibilidade geográfica;

g) ampliação da facilidade de acesso a medicamentos; e

h) possibilidade de garantia da disponibilidade de medicamentos nas farmácias privadas. 
Reforça-se ainda que a estratégia da parceria do governo federal com o setor privado varejista farmacêutico, com a utilização dos pontos de distribuição dos medicamentos essenciais já estruturados, pareceu não apenas apresentar benefícios de tempo e velocidade para aceleração da ampliação do acesso, mas também de redução de custos para o Estado. A meta inicial do governo federal era construir mais de 20 mil farmácias populares (rede própria) em todas as unidades federativas do país, porém em dois anos construiu apenas 250 . O custo de construção e operacionalização neste eixo, uma vez que envolvem a gestão do município, são maiores para o governo federal. Estes valores inexistem com a parceria das farmácias privadas. Este é um benefício reconhecido.

A estratégia do PAFP mostrou-se ser mais econômica que a da rede Fiocruz, embora não seja o Estado o comprador direto dos medicamentos. Uma justificativa possível para o crescimento acelerado dos convênios firmados, demonstrando ser este eixo uma ação prioritária para o Estado. Entretanto, há algumas limitações a serem consideradas para que o PAFP tenha melhor desempenho no que se propõe, em especial em relação à dimensão do poder de compra da farmácia privada.

Neste sentido, uma alternativa para que o governo atue, melhorando o poder de compra das farmácias conveniadas ao PAFP, é regular práticas de preços junto aos fornecedores farmacêuticos através de sistema de pregão e de licitações, semelhante à prática da rede própria, gerida pela Fiocruz. Isto permitiria maior transparência do processo com controle e regulação do governo federal e ainda a redução dos gastos governamentais.

Tendo em vista que os principais resultados desejados do acesso a medicamentos são o uso racional e a satisfação do usuário com o serviço de saúde e estes são os elementos centrais do modelo de acesso a medicamentos proposto pela WHO e MSH (2000), sugere-se como estudo futuro um acompanhamento mais direto com os usuários do PAFP, possibilitando avaliar se estes objetivos estão sendo alcançados.

Conclui-se que cabe ao Estado não apenas a criação de políticas públicas, mas a sistemática de acompanhamento e controle das mesmas. Percebe-se que as políticas voltadas à criação de Programas para a ampliação do acesso aos medicamentos essenciais são fundamentais para melhoria dos indicadores sociais, de crescimento e desenvolvimento econômico do país. É necessário, no entanto, quando da elaboração dessas políticas que um modelo seja adotado e suas dimensões avaliadas. Este deve sintetizar a dinâmica que envolve uma complexa rede tanto de atores públicos e privados, em seus diferentes papéis, quanto de diferentes intervenções com suas dimensões e determinantes sociais e políticos.

ABSCRAT: This paper analyses the structure of the "Farmácia Popular do Brasil" Program (PFPB), based on the proposed dimensions of the World Health Organization (OMS) model together with the Management Sciences for Health (MSH). It discusses concepts and approaches of the State role on the perspective of the economic development, access to medicines models and health sector indicators as part of economic development in Brazil. While featuring the PFPB, aligned elements have been identified to the dimensions availability, accessibility, acceptability, and affordability. The strategy of "Aqui tem Farmácia Popular" (PAFP) showed to be economic, justifying that it is kept as the State priority strategy. Although, there are limits to be considered in order to have a better performance in what it is proposed, specially related to the private pharmacy affordability dimension.

KEY-WORDS: Economic Development, Access to Essential Medicaments, Aqui tem Farmácia Popular Program. 


\section{REFERÊNCIAS}

BERMUDEZ, J.; et alii. (2004). Acceso a medicamentos: derecho fundamental, papel del Estado. ENSP, Rio de Janeiro.

BIGDELI, M.; et alii. (2012). Access to medicines from a health system perspective. Health Policy and Planning,7:692-704, Oxford.

BRASIL. (1988). Constituição da República Federativa do Brasil. Diário Oficial da República Federativa do Brasil, Brasília.

BRASIL. (1990). Lei $n^{o}$ 8.080. Diário Oficial da Republica Federativa do Brasil, Brasília. Disponível em: Erro! A referência de hiperlink não é válida.

BRASIL. (1998). Instrução Normativa SRF $n^{\circ}$ 162. Diário Oficial da República Federativa do Brasil, Brasília, 4:5.

BRASIL. (2008). Avaliação econômica em saúde: desafios para gestão no Sistema Único de Saúde. Editora do Ministério da Saúde, Brasília.

BRASIL. (2013). Financiamento da saúde: Brasil e outros países com cobertura universal. Câmara dos Deputados. Consultoria de Orçamento e Fiscalização Financeira - CONOF/CD, Brasília. Disponível em: http://www2.camara.leg. br/atividadelegislativa/orcamentobrasil/estudos/2013/NTn12de2013 Financia mentodaSadeBrasileOutrosPasesV.Prel.. pdf.

BRASIL. (2014). Em três anos, 19 milhões de brasileiros foram beneficiados pelo saúde não tem preço. Editora do Ministério da Saúde, Brasília. Disponível em: http://www.blog.saude.gov.br/index.php/programasecampanhas/33644mais-de-19-milhoes-de-brasileiros-foram-beneficiados-com-medicamentos-de-graca-em-tres-anos.

CONSELHO NACIONAL DOS SECRETÁRIOS DE SAÚDE - CONASS. (2003). A saúde na opinião dos brasileiros: prógestores. Brasília.

CORAZZA, G. (1985). Teoria econômica e Estado: (de Quesnay a Keynes). Faculdade de Ciências Econômicas, Universidade Federal do Rio Grande do Sul, Porto Alegre.

DONABEDIAN, A. (1984). La calidad de la atención médica: definicón e métodos de evaluación. La Prensa Médica Mexicana, México.

FROST, L. J.; REICH, M. R. How do good health technologies get to poor people in poor countries? Boston: Harvard Center for Population and Development Studies, 2010.

FUNDAÇÃO OSWALDO CRUZ - Fiocruz. (2011). Prestação de contas ordinária anual relatório de gestão do exercício de 2011. Disponível em: http://www.Fiocruz.br/acessoainformacao/media/Relatorio_gestao_2011.pdf

FUNDAÇÃO OSWALDO CRUZ - Fiocruz \& et. alii. (2012). A saúde no Brasil em 2030: diretrizes para a prospecção estratégica do sistema de saúde brasileiro. FIOCRUZ, Rio de Janeiro. Disponível em: https://portal.fiocruz.br/sites/ portal.fiocruz.br/files/documentos/miolo_saude_brasil_2030.pdf. 
INSTITUTO BRASILEIRO DE GEOGRAFIA E ESTATÍSTICA - IBGE. (2012). Conta-Satélite de Saúde: Brasil 2007-2009. Rio de Janeiro. Disponível em: http://www.ibge.gov.br/home/estatistica/economia/economia_saude/css_2007_2009/ economia_saude.pdf.

KEYNES, J. (1936). Teoria geral do emprego, do juro e da moeda. Fundo de Cultura, Rio de Janeiro.

KEYNES, J. (2004). The end of laissez-faire. Prometheus Books, New York.

LUIZA, V. (2003). Acesso a medicamentos no Estado do Rio de Janeiro.Fundação Oswaldo Cruz, Rio de Janeiro.

MADRID, Y., VELÁSQUEZ, G. \& FEFER, H. (1998). Pharmaceuticals and bealth sector reform in the Americas: an economic perspective. OPS, Washington.

MANAGEMENT SCIENCE FOR HEALTH - MSH. International Drug price indicator guide. 2004. Disponível em: $<$ http://erc.msh.

org $/$ mainpage.cfm?file $=1.0$. htm\&id $=1 \&$ temptitle $=$ Introduction $\&$ module $=$ DMP\&language $=$ English $>$. Acesso em: 28 nov. 2012.

MÉDECINS SANS FRONTIERES - MSF. (2002). A pricing guide for the purchase of ARVs for developing countries: untangling the of price reductions. Genève.

MYRDAL, G. (1968). Teoria econômica e regiões subdesenvolvidas. Saga, Rio de Janeiro.

PAINA, L. \& PETERS, D. (2011). Understanding pathways for scaling up health services through the lens of complex adaptive systems. Health Policy and Planning. Oxford, 1-9.

PENCHANSKY, D. \& THOMAS, J. (1981). The concept of access: definition and relationship to consumer satisfaction. Medical Care. Philadelphia, 2:127-140.

PEREIRA, L. (1996). Trabalho apresentado ao seminário sobre Reforma do Estado na América Latina organizado pelo Ministério da Administração Federal e Reforma do Estado e patrocinado pelo Banco Interamericano de Desenvolvimento. Revista do Serviço Público. Brasília, 1.

RÊGO, E. (2000). Políticas de regulação do mercado de medicamentos: a experiência internacional. Revista do BNDES. Rio de Janeiro, 7:367-400.

REIS, A. L. A.; BERMUDEZ, J. Aspectos econômicos: mercado farmacêutico e preços de medicamentos. In: BERMUDEZ, J.; OLIVEIRA, M. A.; ESHER, A. (Org.). Acesso a medicamentos. Rio de Janeiro: Escola Nacional de Saúde Pública, Fiocruz, 2004. p. 139-55.

RODRIK, D. (2005). Growth strategies. In: AGHION, P. \& DURLAUF, S. Handbook of Economic Growth. Elsevier, Oxford, 1:967-1014.

SILVA FILHO, H. (2010). Direito à saúde: a inconstitucionalidade do Programa Farmácia Popular do Brasil. Departamento de Ciências Jurídicas, Centro Universitário de João Pessoa, João Pessoa.

STYLO FARMA. Programa Farmácia Popular do Brasil contabiliza mais de 20 mil pontos de distribuição de medicamento. 2012. Disponível em: <www.stylofarma.com.br/programa_farmacia_popular_do_brasil_ contabiliza_mais_de20m >. Acesso em: 10 fev.2014. 
THE WORLD BANK - TWB. (2014). World Development Indicators. Disponível em: < http://data.

world bank.org/data-catalog/world-development-indicators $>$.

VIANA, A. \& ELIAS, P. (2007). Saúde e desenvolvimento. Ciência saúde coletiva. Rio de Janeiro, 12:1765-1777.

Disponível em: $<$ http://www.scielo.br/scielo.php?script $=$ sci_arttext\&pid $=$ S1413-81232007000700002\&lng $=$ en $\&$ $\mathrm{nrm}=$ iso $>$.

WORLD HEALTH ORGANIZATION - WHO. (1946). Constituição da Organização Mundial da Saúde (OMS/WHO). Disponível em: < http://www.direitoshumanos.usp.br/index.php/OMS-Organiza \%C3\%A7\%C3\%A3o-Mundial-daSa\%C3\%BAde/constituicao-da-organizacao-mundial-da-saude-omswho.html > .

WORLD HEALTH ORGANIZATION - WHO. (2000). Medicines Strategy: Framework for action in essential drugs and medicines policy 2000-2003. Geneva.

WORLD HEALTH ORGANIZATION - WHO. (2001). Macroeconomics and bealth: investing in bealth for economic development. Report of the Commission on Macroeconomics and Health, Geneva.

WORLD HEALTH ORGANIZATION - WHO. (2004). WHO medicines strategy: Countries at the Core: 2004-2007. Geneva.

WORLD HEALTH ORGANIZATION - WHO; MANAGEMENT SCIENCES FOR HEALTH - MSH. (2000). Defining and measuring access to essential drugs, vaccines, and health commodities. Disponivel em: <http://www.msh.org/ seam/reports/Access_Meeting_Ferney_Voltaire_1.pdf $>$.

WORLD HEALTH ORGANIZATION - WHO; WORLD TRADE ORGANIZATION SECRETARIATS - WTO. (2001). Report of the workshop on differential pricing and financing of essential drug. Høsbjør. 